\title{
Circular Polarized Carbon-NanotubePatch Antenna embedded in Superstrates Anisotropic Media
}

\author{
Mamdoh Gharbi ${ }^{1}$, Adnan Affandi $^{1}$, Abdullah Dobaie $^{1}$ \\ ${ }^{I}$ (Department of Electrical and Computer, Engineering/ King Abdul Aziz University, Saudi Arabian)
}

\begin{abstract}
The current distribution on surface of the Patch Antenna is radiating in the presence of the Superstrates layered anisotropic medium. The electric and magnetic field in the upper half-space are formulated using Dyadic Green Function in terms of Sommerfeld-Weyl-type integrals. The stationary phase method is used to obtain far-field expressions. Carbon-Nanotube Composite (CNT) as the radiating element for Star Patch Antenna is used. .Return loss, Axial Ratio ( $<3$ dB Circular Polarized), Gain and Directivity parameters of Carbon-Nanotube Star Patch Antenna are compared for different anisotropic materials.
\end{abstract}

Keywords: Anisotropic, patch antenna, Moment Method, Dyadic Green Function. Carbon-Nanotube Composite

\section{Introduction}

Analyzing the antenna parameters with anisotropic media was appeared in many works [1-4]. The affect of anisotropic ratio on the far-field radiation pattern was investigated ([5]. Because of cost, weight, fabrication procedure it limited the use of copper or metals for patch or ground plane. Several composite materials as replacement for metals were studied in many recent studies [6-8]. Due to electrical properties and favorable mechanical Carbon Nanotube Composites have been used for electromagnetic applications [912].In this study, using the dyadic Green's function formulation the electric and magnetic fields in the upper half-space due to the current distribution on the Star Patch are obtained [13]. The Sommerfeld-Weyl-type integrals of electric and magnetic fields are calculated in the radiation zone or far-field region [13].

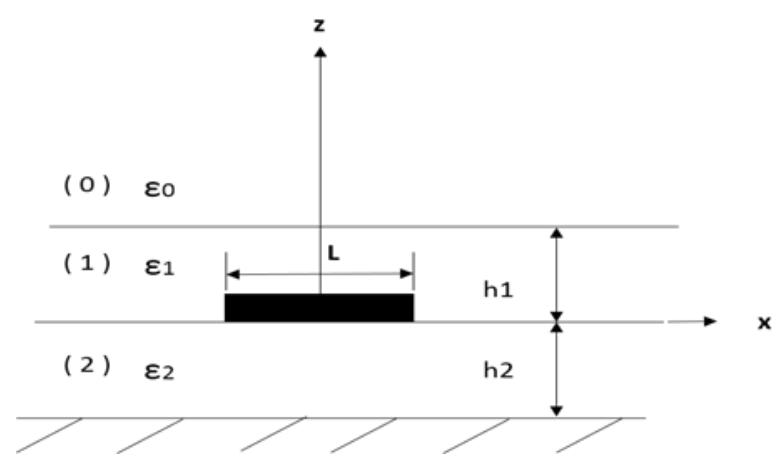

Fig. 1. Geometry of the Problem with patch in region (1) and observation point in region (0)

\section{Expressing The Electric and Magnetic Fields}

\subsection{Electric and Magnetic Fields in the Upper-Half-space}

In the upper-half space (region 0 ), the far-field satisfies the following relations:

$$
\begin{aligned}
E_{0 \theta}=-\frac{E_{0 z}}{\sin \theta} & \\
& H_{0 \phi}=\frac{1}{\eta_{0}} E_{0 \theta}
\end{aligned}
$$

for TM waves, and

$$
\begin{aligned}
& H_{0 \theta}=-\frac{H_{0 \mathrm{z}}}{\sin \theta} \\
& E_{0 \phi}=-\eta_{0} H_{0 \theta}
\end{aligned}
$$

for TE waves, and 
$\eta_{0}=\sqrt{\frac{\mu_{0}}{\varepsilon_{0}}}$

The electric field $E_{0 z}$ can be obtained as

$E_{0 z}=\hat{z} \cdot \bar{E}_{0}=\hat{z} \cdot i \omega \iint_{S^{\prime}} d r^{\prime} \overline{\bar{G}}_{01}\left(\bar{r}, \bar{r}^{\prime}\right) \cdot \bar{J}_{s}\left(\bar{r}^{\prime}\right)=i \omega \iint_{S^{\prime}} d x^{\prime} d y^{\prime} G_{01}^{(z, x)}\left(\bar{r}, \bar{r}^{\prime}\right) J_{x}\left(x^{\prime}, y^{\prime}\right)$

where

$$
G_{01}^{(z, x)}\left(\bar{r}, \bar{r}^{\prime}\right)=\hat{z} \cdot \overline{\bar{G}}_{01}\left(\bar{r}, \bar{r}^{\prime}\right) \cdot \hat{x}
$$

$\overline{\bar{G}}_{01}\left(\bar{r}, \bar{r}^{\prime}\right)$ can be obtained as shown in [13] ( See Fig. 1). For anisotropic medium:

$$
G_{01}^{(z, x)}\left(\bar{r}, \bar{r}^{\prime}\right)=\iint_{-\infty}^{\infty} d k_{x} d k_{y} e^{i \bar{k}_{s} \cdot\left(\bar{r}_{s}-\bar{r}_{s}^{\prime}\right)} g_{01}^{(z, x)}\left(\bar{k}_{s}, z, z^{\prime}\right)
$$

where

$$
g_{01}^{(z, x)}\left(\bar{k}_{s}, z\right)=\frac{-i \mu_{0}}{8 \pi^{2}} \frac{r_{1}^{T M}}{k_{0}} X_{\cup 0,1}^{T M} e^{i k_{0 z} z} e^{i k_{1 z}^{(e)} h_{1}} \frac{k_{x}}{k_{1}}\left(1-R_{\cap 1}^{T M} e^{i k_{12}^{(e)} h_{1}}\right)
$$

where $z^{\prime}$ is substituted by $\left(-h_{1}\right)$.

Thus, the $z$-component of the electric field in region (0) is given by

$$
E_{0 z}=(i) \frac{\omega \mu_{0}}{4 \pi} \cdot \iint_{S^{\prime}} d x^{\prime} d y^{\prime} J_{x}\left(x^{\prime}, y^{\prime}\right) \cos \phi \cdot \int_{0}^{\infty} d k_{\rho} k_{\rho}^{2} g_{E}\left(k_{\rho}\right) e^{i k_{0 z} z} J_{1}\left(k_{\rho} \mid \bar{\rho}-\bar{\rho}^{\prime}\right)
$$

as Sommerfeld-type integral [12].

The magnetic field in the upper half-space can be obtained from Maxwell's equations

$$
\bar{H}_{0}=\frac{1}{i \omega \mu_{0}} \nabla \times \bar{E}_{0}
$$

That is

$$
\bar{H}_{0}(\bar{r})=\frac{1}{\mu_{0}} \iint_{S^{\prime}} d x^{\prime} d y^{\prime} \nabla \times \overline{\bar{G}}_{01}\left(\bar{r}, \bar{r}^{\prime}\right) \cdot \bar{J}_{s}\left(x^{\prime}, y^{\prime}\right)
$$

where

$\overline{\overline{\mathrm{G}}}_{01}\left(\bar{r}, \bar{r}^{\prime}\right)=$

$\frac{i \mu_{0}}{8 \pi^{2}} \iint_{-\infty}^{\infty} d \bar{k}_{s} e^{i \bar{k}_{s} \cdot\left(\bar{r}_{s}-\bar{r}_{s}^{\prime}\right)} e^{i k_{0 z} z}\left\{\frac{r_{1}^{T E}}{k_{1 z}^{(h)}} X_{\cup 0,1}^{T E} e^{i k_{1 z}^{(h)} h_{1}} \hat{h}\left(k_{0 z}\right)\left[\hat{h}\left(k_{1 z}^{(h)}\right)+R_{\cap l}^{T E} e^{i k_{1 z}^{(h)} h_{1}} \hat{h}\left(-k_{1 z}^{(h)}\right)\right]\right\}+$

$\left.\frac{r_{1}^{T M}}{k_{1 z}^{(e)}} X_{\cup 0,1}^{T M} e^{i k_{1 z}^{(e)} h_{1}} \hat{v}\left(k_{0 z}\right)\left\lfloor\hat{v}\left(k_{1 z}^{(e)}\right)+R_{\wedge 1}^{T M} e^{i k_{1 z}^{(e)} h_{1}} \hat{v}\left(-k_{1 z}^{(h)}\right)\right]\right\}$

Hence, the magnetic field $H_{0 z}$ can be obtained as

$H_{0 z}=\frac{-1}{4 \pi} \iint_{S^{\prime}} d x^{\prime} d y^{\prime} J_{x}\left(x^{\prime}, y^{\prime}\right) \sin \phi \cdot \int_{0}^{\infty} d k_{\rho} k_{\rho}^{2} g_{H}\left(k_{\rho}\right) e^{i k_{0 z} z} \cdot J_{1}\left(k_{\rho}\left|\bar{\rho}-\bar{\rho}^{\prime}\right|\right)$

as Sommerfeld type integral.

\subsection{Far-Field Expressions}

The $z$ - component of the electric field in the upper half-space given by Eq. 2.10, can be written as

$E_{0 z}(\bar{r})=\frac{i \omega \mu_{0}}{4 \pi} \iint_{S^{\prime}} d x^{\prime} d y^{\prime} J_{x}\left(x^{\prime}, y^{\prime}\right) \cos \phi I_{E}$

where

$I_{E}=\int_{0}^{\infty} d k_{\rho} k_{\rho}^{2} g_{E}\left(k_{\rho}\right) e^{i k_{0 z} z} J_{1}\left(k_{\rho}\left|\bar{\rho}-\bar{\rho}^{\prime}\right|\right)$ 
which can be put in the form

$I_{E}=\frac{1}{2} \int_{-\infty}^{\infty} d k_{\rho} k_{\rho}^{2} g_{E}\left(k_{\rho}\right) e^{i k_{0 z} z} H_{1}^{(1)}\left(k_{\rho}\left|\bar{\rho}-\bar{\rho}^{\prime}\right|\right)$

Using the large argument approximation for the Hankel function, the rapidly varying part of the integrand in Eq.

2.17 is asymptotic to the phase factor $e^{i k_{\rho}\left|\bar{\rho}-\bar{\rho}^{\prime}\right|+i k_{0 z} z}$. Thus, the location of the stationary phase point is given by

$\left.\frac{\partial}{\partial k_{\rho}} i\left(k_{\rho}\left|\bar{\rho}-\bar{\rho}^{\prime}\right|+k_{0 z} z\right)\right|_{k_{\rho 0}}=0$

The solution is given by

$k_{\rho_{0}}=k_{0} \sin \theta, \quad k_{0 z 0}=k_{0} \cos \theta$

with $\theta=\sin ^{-1} \frac{\left|\bar{\rho}-\bar{\rho}^{\prime}\right|}{\left|\bar{r}-\bar{\rho}^{\prime}\right|}$, (see Fig. 2.2).

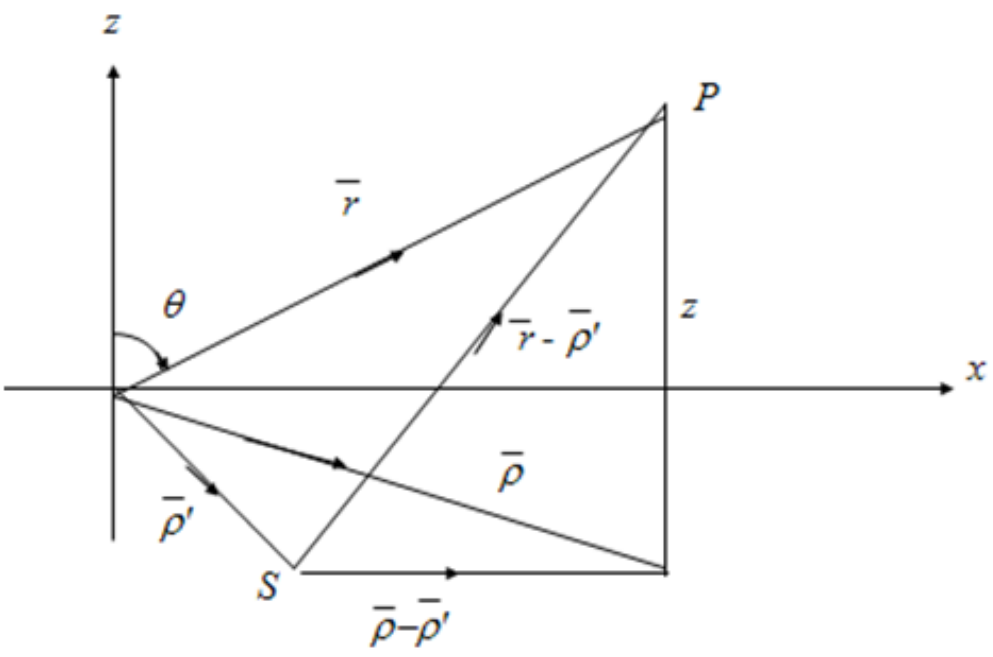

Fig. 2, Relation between $\rho$ and $\theta$

This leads to the following expression for $I_{E}$

$I_{E} \sim-i g_{E}\left(k_{\rho_{0}}\right) k_{\rho_{0}} e^{-i \pi / 2} k_{0 z 0} \frac{e^{i k_{0}\left|\bar{r}-\bar{\rho}^{\prime}\right|}}{\left|\bar{r}-\bar{\rho}^{\prime}\right|} \quad, r \rightarrow \infty$

But

$\left|\bar{r}-\bar{\rho}^{\prime}\right| \cong r-\hat{r} \cdot \bar{\rho}^{\prime}$,

thus

$I_{E} \sim k_{0}^{2} \sin \theta \cos \theta g_{E}\left(k_{\rho_{0}}\right) e^{-i \bar{k}_{0} \cdot \bar{\rho}^{\prime}} \frac{e^{i k_{0} r}}{r}, r \rightarrow \infty$

Thus,

$E_{0 z}(\bar{r}) \sim \frac{-i \omega \mu_{0}}{4 \pi} k_{0}^{2} \sin \theta \cos \theta \cos \Phi g_{E}\left(k_{\rho_{0}}\right) \frac{e^{i k_{0} r}}{r} \times \iint d x^{\prime} d y^{\prime} J_{x}\left(x^{\prime}, y^{\prime}\right) e^{-i \bar{k}_{0} \cdot \bar{\rho}^{\prime}}$

Following similar procedure, we can get for the magnetic field in the upper half-space as $r \rightarrow \infty$, the following expression

$H_{0 z}(\bar{r}) \sim \frac{i}{4 \pi} k_{0}^{2} \sin \theta \cos \theta \sin \Phi g_{H}\left(k_{\rho_{0}}\right) \frac{e^{i k_{0} r}}{r} \times \iint d x^{\prime} d y^{\prime} J_{x}\left(x^{\prime}, y^{\prime}\right) e^{-i \bar{k}_{0} \cdot \bar{\rho}^{\prime}}$

Thus, using Eq. 2.1 - Eq. 2.4, the field patterns are readily obtained 


\section{Results And Discussion}

CNT Star patch numerical results embedded in anisotropic superstrate structures are presented. Figure 2, shows the geometry of CNT Star Patch Antenna. The effects of anisotropy of Superstrate layers in Sparameters, Axial Ratio, Directivity and gain are investigated. To simulate the Far Field Radiation of CNT Star Patch, a Matlab program is written. For Validation propose simulation software CST STUDIO SUITE® is used. The compered result between the Two programs was good similarity. A microstrip CNT Star patch of length $L p=42.12 \mathrm{~mm}$ and width $\mathrm{W}=42.12 \mathrm{~mm}$ on the top of RTDURIOD 5870 an isotropic substrate of permittivity $\varepsilon \mathrm{r}=2.33$, and thickness $\mathrm{hs}=1.5 \mathrm{~mm}$. The Ground plate of square shape with length $\mathrm{L}=52 \mathrm{~mm}$. The CTN Star patch is fed with coaxial probe. The following anisotropic substrates are used: pirolytic boron nitride, or pbn $\varepsilon_{x x} \square=5.12 ; \varepsilon_{z z} \square=3.4$ ), sapphire $\left(\varepsilon_{x x} \square=9.4 ; \varepsilon_{z z} \square=11.6\right.$ ), Epsilam $10\left(\varepsilon_{x x} \square=13 ; \varepsilon_{z z} \square=10.6\right)$. For $\varepsilon_{y}=1$ (vacuum or Air) use Foam flakes $\varepsilon_{y}=1.1$, which is good approximation.

\subsection{Input Impedance of Star patch with superstrate anisotropic layer}

The Anisotropic Superstrate effect for CNT Star Patch Antenna is conducted.The Superstrate Layer assumed to be anisotropic media with permittivity pirolytic boron nitride ( pbn) $\varepsilon_{z x}=5.12 ; \varepsilon_{z z}=3.4$. The EPlane Radiation of CNT Star Patch antenna fed by Coaxial Cable was shown in Fig. 3.

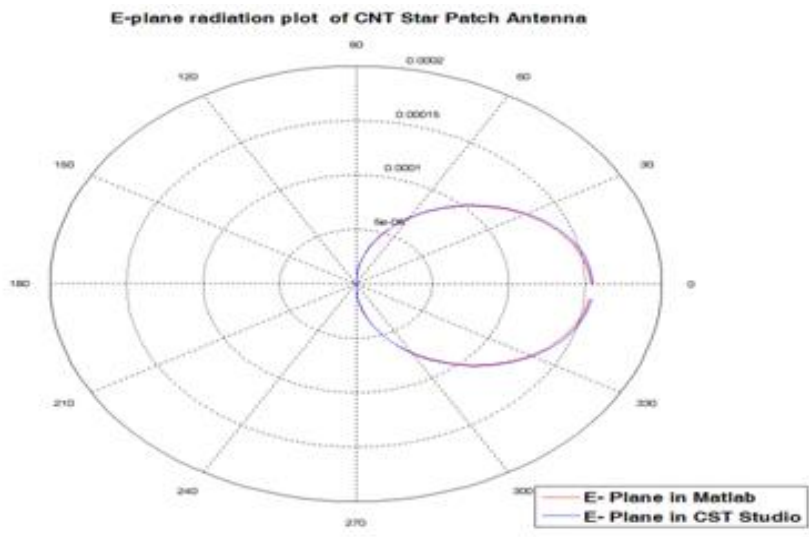

Fig. 3, E - Plane radiation of CNT Star Patch Antenna

The CST Simulating result almost identical to the theoretical model.

3.2 Farfield Directivities and gainsof CNT Star patch without any Superstrate

A CNT Star Patch without any Superstrate is considered. Figure 4,show, the circuit of CNT Star patch.

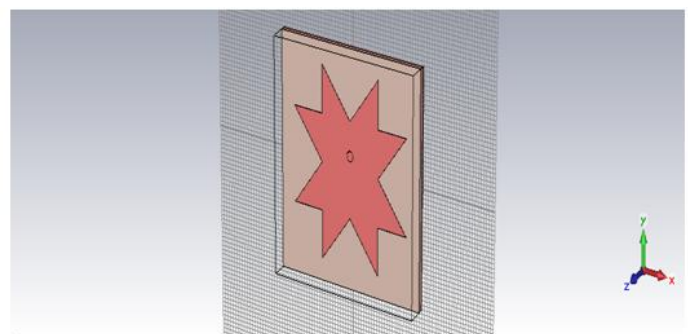

Fig. 4, CNT Star Patch Antenna

Table 1, Directivities and Gains of CNT Star Patch Antenna without Superstrate

\begin{tabular}{|c|c|c|}
\hline $\begin{array}{c}\mathrm{F} \\
\mathrm{GHz}\end{array}$ & $\begin{array}{c}\text { Directivity } \\
\mathrm{dBi}\end{array}$ & $\begin{array}{c}\text { Gain } \\
\mathrm{dBi}\end{array}$ \\
\hline 5.5 & 3.95 & 2.11 \\
\hline 8.5 & 5.94 & 2.48 \\
\hline 10.5 & 3.65 & 0.442 \\
\hline 12.5 & 1.39 & -0.512 \\
\hline 14.5 & 4.49 & 1.49 \\
\hline 17.5 & 4.54 & 2.3 \\
\hline 19.5 & 4.96 & 3.02 \\
\hline 21.5 & 3.19 & 1.69 \\
\hline 23.5 & 4.51 & 2.71 \\
\hline 25.5 & 9.53 & 8.06 \\
\hline
\end{tabular}


Farfield Directivity Abs (Phi=90)

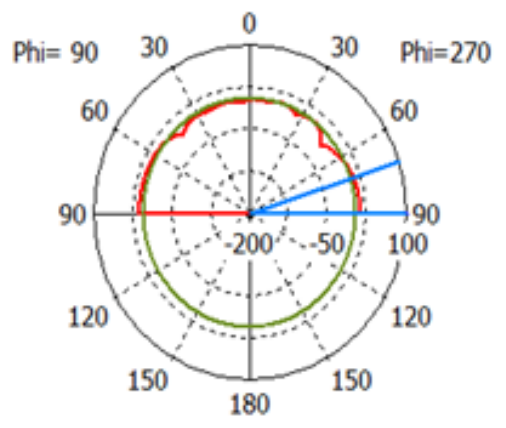

Theta / Degree vs. dBi
— farfield $(f=25.5)[1]$

\author{
Frequency $=25.5$ \\ Main lobe magntude $=11.2 \mathrm{~dB}$ \\ Main lobe direction $=90.0 \mathrm{deg}$. \\ Angular width $(3 \mathrm{~dB})=17.6 \mathrm{deg}$. \\ Side lobe level $=-5.9 \mathrm{~dB}$
}

Fig. 5. Directivity of CNT Star Patch at $\mathrm{Fr}=25.5 \mathrm{GHz}$

Farfield Gain Abs (Phi=90)

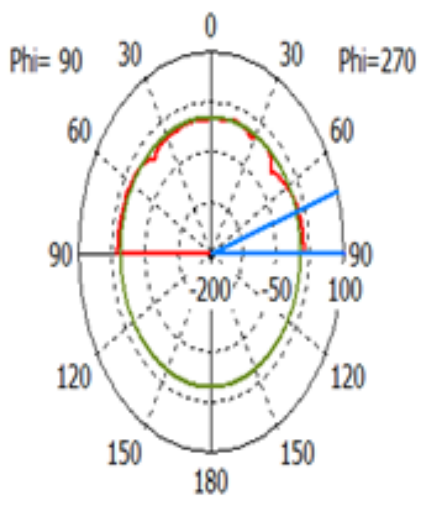

Theta / Degree vs. dB farfield $(f=25.5)[1]$
Frequency $=25.5$

Man lobe magntude $=9.69 \mathrm{~dB}$

Main lobe drection $=90.0 \mathrm{deg}$.

Angular with $(3 \mathrm{~dB})=17.6 \mathrm{deg}$.

Side lobe level $=-5.9 \mathrm{~dB}$

Fig. 6. Gain of CNT Star Patch at $\mathrm{Fr}=25.5 \mathrm{GHz}$

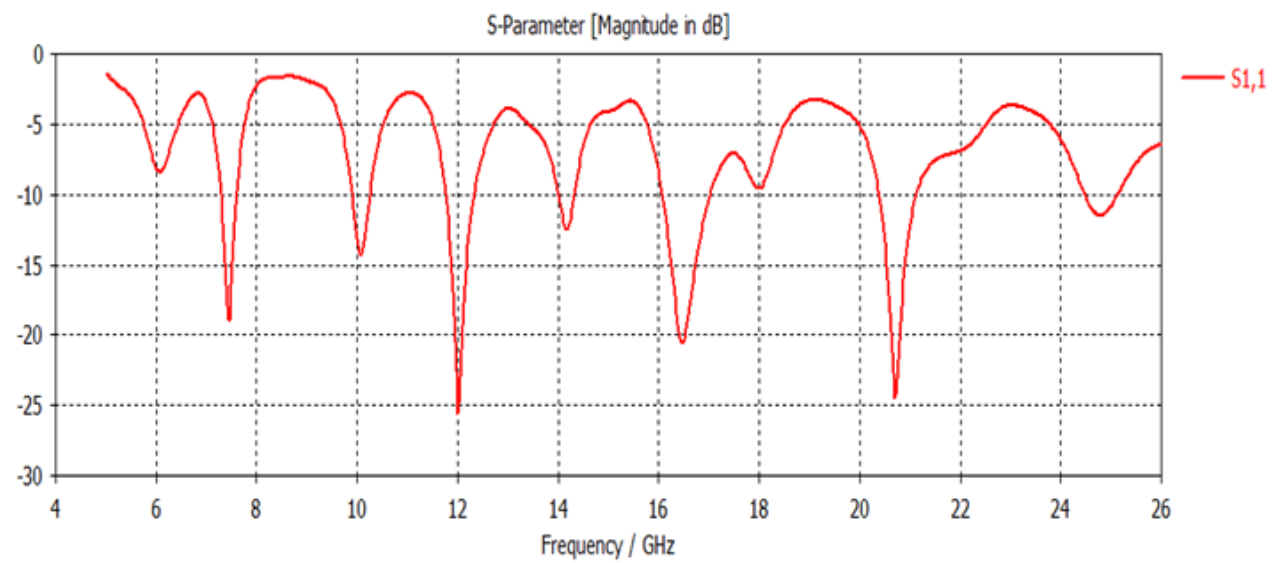

Fig. 7, S11 of CNT Star Patch Antenna

Design Criteria's for CNT Star Patch Antenna are :

1) $\mathrm{S} 11 \leq-10 \mathrm{~dB}$

2) Axial Ratio $\leq 3 \mathrm{~dB}$

3) Gain should be maximum

4) Directivity should be maximum 
Table 2, S11, Axial Ratio, Gain and Directivities for best Design of CNT Star Patch Antenna without Superstrate

\begin{tabular}{|c|c|c|c|c|}
\hline $\begin{array}{c}\text { Frequency } \\
\text { GHz }\end{array}$ & $\begin{array}{c}\text { S11 } \\
\mathbf{d B}\end{array}$ & $\begin{array}{c}\text { Axial Ratio } \\
\mathbf{d B}\end{array}$ & $\begin{array}{c}\text { Gain } \\
\mathbf{d B}\end{array}$ & $\begin{array}{c}\text { Directivity } \\
\mathbf{d B}\end{array}$ \\
\hline 7.517 & -22.75 & 6.3 & 4.4 & 5.53 \\
\hline 12.123 & -16.6 & 8.02 & 3.79 & 4.43 \\
\hline 14.279 & -18.47 & 0.661 & 4.52 & 5.75 \\
\hline 16.582 & -27.19 & 1.456 & 8.66 & 9.26 \\
\hline 18.15 & -11.52 & 3.49 & 6.32 & 7.29 \\
\hline 20.894 & -24.04 & 1.099 & 3.7 & 3.94 \\
\hline 24.961 & -13.32 & 2.704 & 8.68 & 8.95 \\
\hline
\end{tabular}

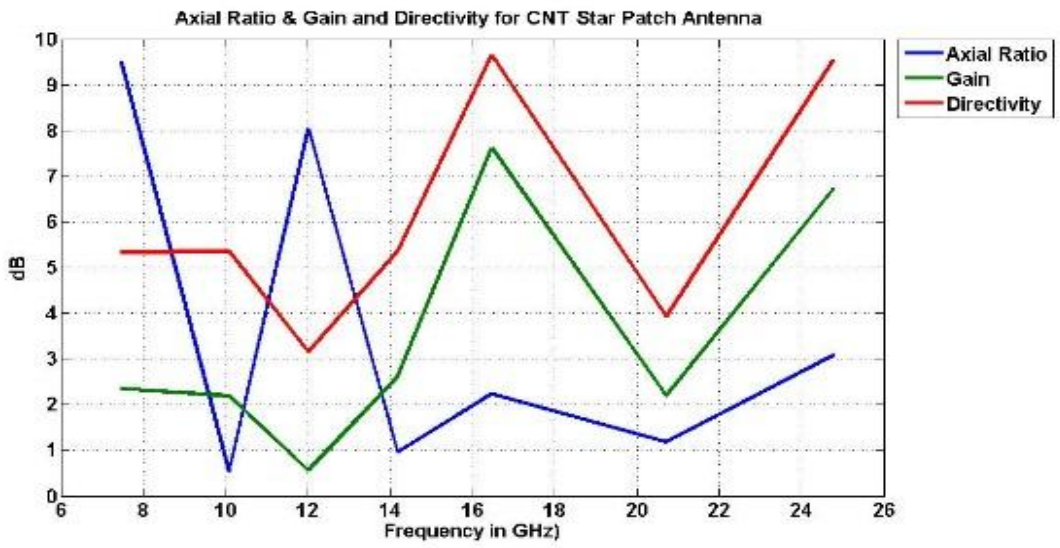

Fig. 8, Axial Ratio, Gain and Directivities for best Design of CNT Star Patch Antenna without Superstrate

CST Studio Suite designs the CNT Sstar patch (Fig. 4). Fig. 7. Shows S11 for this configuration. It is clear from figure 5, star patch directivity is $11.2 \mathrm{dBi}$. The gain of that patch is $9.69 \mathrm{dBi}$ as shown in Fig. 6 .

From Table 2 and Figure 8, the best Design parameters are :

$\mathrm{F}=16.582 \mathrm{GHz}$

$\mathrm{S} 11=-27.19 \mathrm{~dB}$

Axial Ratio $=1.456 \mathrm{~dB}$

Gain $=8.65 \mathrm{~dB}$

Directivity $=9.26 \mathrm{~dB}$

\subsection{Farfield Directivities and gainsof CNT Star patch with one Superstrate layer}

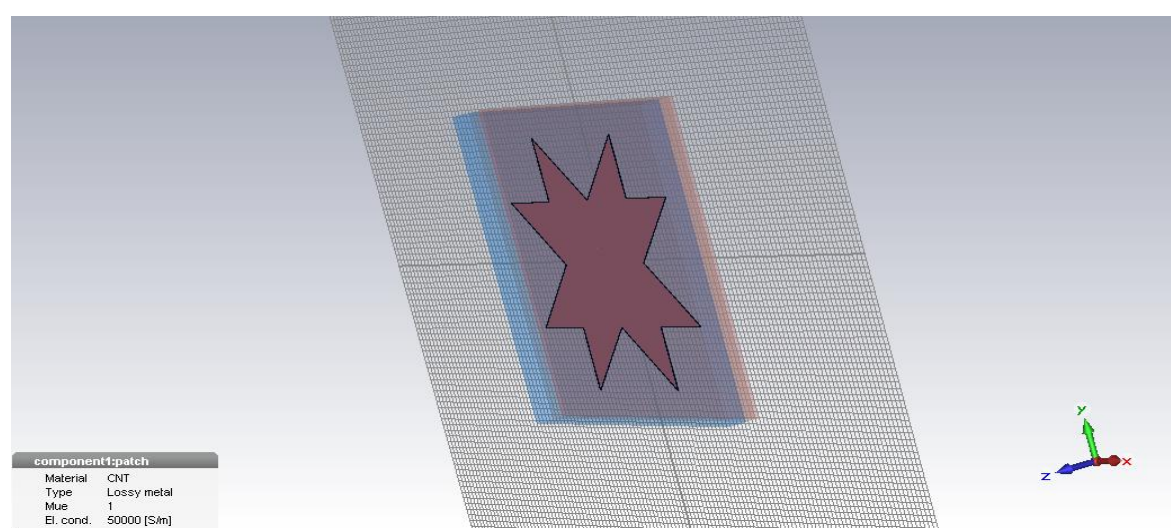

Fig. 9. CNT Star Patch Antenna with one anisotropic superstrate Layer, $\varepsilon_{x x}=5.12 ; \varepsilon_{z z}=3.4$ 


\begin{tabular}{|c|c|c|}
\hline $\mathrm{F}$ & $\begin{array}{c}\text { Directivity } \\
\mathrm{dBi}\end{array}$ & $\begin{array}{c}\text { Gain } \\
\mathrm{dBi}\end{array}$ \\
\hline 1.5 & 4.54 & -2.46 \\
\hline 2.5 & 7.09 & -1.36 \\
\hline 4.5 & 4.3 & 2.23 \\
\hline 7.5 & 4.65 & 2.84 \\
\hline 11.5 & 12.2 & 11 \\
\hline 17.5 & 5.94 & 4.86 \\
\hline 19.5 & 5.85 & 4.94 \\
\hline 21.5 & 6.85 & 5.44 \\
\hline
\end{tabular}

Table 3 Directivities and gains of CNT Star Patch Antenna with one Anisotropic Superstrate layer, $\varepsilon_{z x}=5.12$; $\varepsilon_{z z}=3.4$

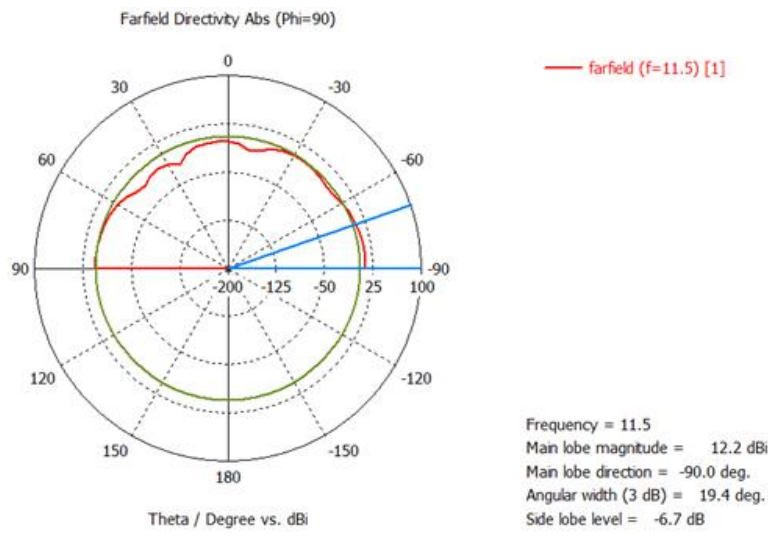

Fig. 10. Directivity of CNT Star Patch at $\mathrm{F}=11.5 \mathrm{GHz}$ with one Anisotropic Superstrate layer, , $\varepsilon_{x x}=5.12 ; \varepsilon_{z z}=$ 3.4

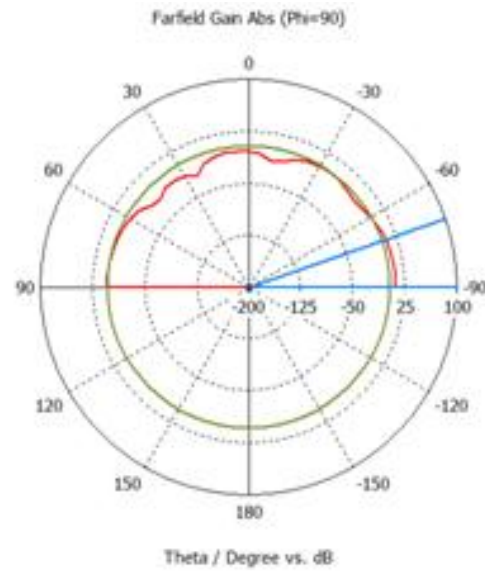

- tarted $(f=11.5)[1]$

Frequency $=11.5$

Man boe magrade - 11 de

Man bbe drection $=-90.0$ deg

Angilar woth $(B \mathrm{~dB})=19.4 \mathrm{deg}$

Side ibe kevel $=-6.7 \mathrm{dg}$

Fig 11. Gain of CNT Star Patch at $\mathrm{F}=11.5 \mathrm{GHz}$ with one Anisotropic Superstrate layer, , $\varepsilon_{z x}=5.12 ; \varepsilon_{z z}=3.4$

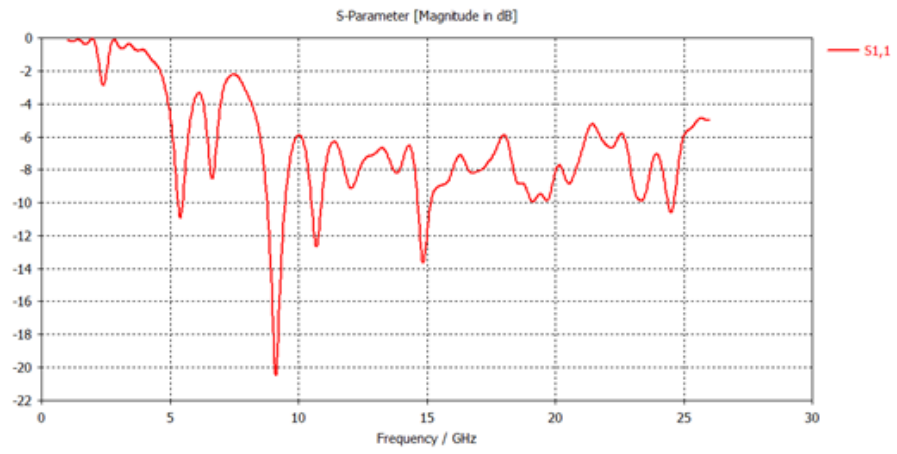

Fig. 12. S11 of CNT Star Patch Antenna with one Anisotropic Superstrate layer, , $\varepsilon_{x x}=5.12 ; \varepsilon_{z z}=3.4$ 
Table 4, S11, Axial Ratio, Gain and Directivities for best Design of CNT Star Patch Antenna with one Anisotropic Superstrate layer, , $\varepsilon_{x x}=5.12 ; \varepsilon_{z z}=3.4$.

\begin{tabular}{|c|c|c|c|c|}
\hline $\begin{array}{c}\text { Frequency } \\
\mathbf{G H z}\end{array}$ & $\begin{array}{c}\text { S11 } \\
\mathbf{d B}\end{array}$ & $\begin{array}{c}\text { Axial Ratio } \\
\mathbf{d B}\end{array}$ & $\begin{array}{c}\text { Gain } \\
\mathbf{d B}\end{array}$ & $\begin{array}{c}\text { Directivity } \\
\mathbf{d B}\end{array}$ \\
\hline 5.399 & -10.89 & 6.03 & 3.47 & 4.99 \\
\hline 9.116 & -20.66 & 3.901 & 4.38 & 5.62 \\
\hline 10.691 & -12.63 & 4.284 & -2.63 & -1.11 \\
\hline 14.849 & -13.63 & 1.574 & 4.48 & 5.60 \\
\hline 24.509 & -10.68 & 4.186 & 7.88 & 8.89 \\
\hline
\end{tabular}

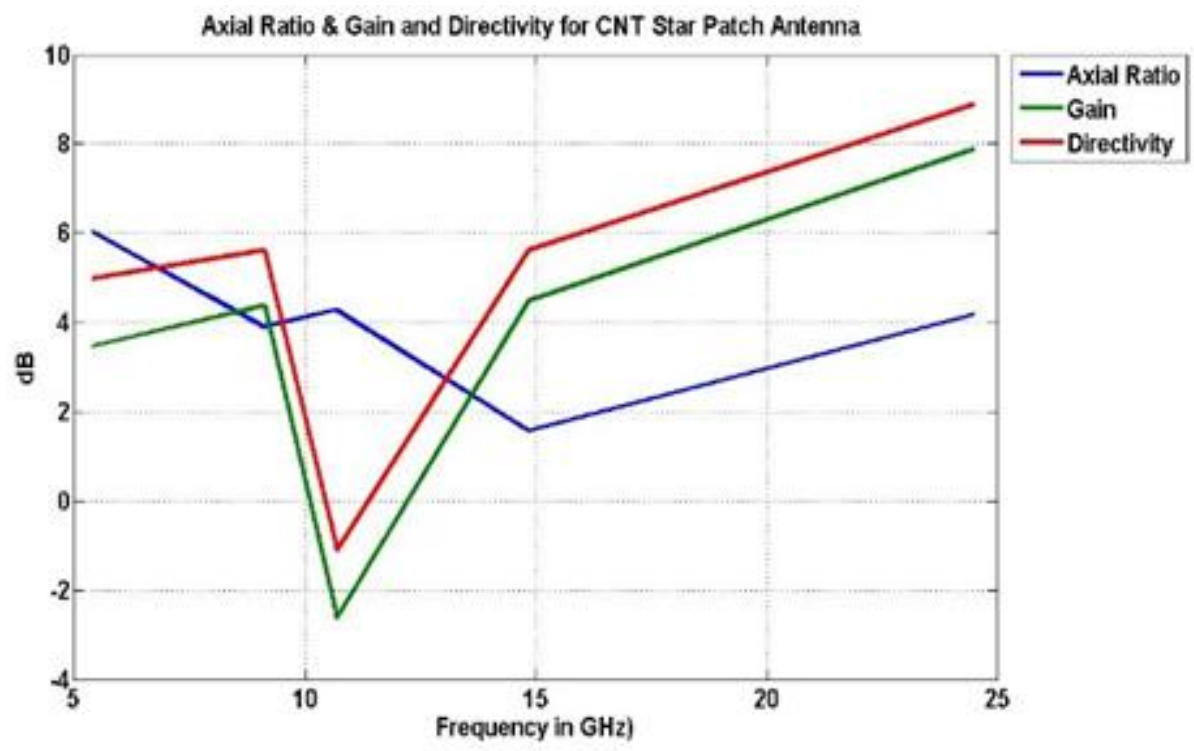

Fig. 13, Axial Ratio, Gain and Directivities for best Design of CNT Star Patch Antenna with one Anisotropic Superstrate layer, $\varepsilon_{x x}=5.12 ; \varepsilon_{z z}=3.4$.

Fig. 9. Shows the Directivity for CNT Star Patch with pirolytic boron nitride, or pbn $\left(\varepsilon_{x z}=5.12 ; \varepsilon_{z z}=3.4\right.$. $)$ Superstrate is greater than CNT Star patch without Superstrate. In addition, the Gain also increased if adding a Anisotropic Superstrate. Fig. 12, shows S11.

From Table 4 and Fig. 13, the best Design parameters are :

$\mathrm{F}=14.849 \mathrm{GHz}$

$\mathrm{S} 11=-13.63 \mathrm{~dB}$

Axial Ratio $=1.574 \mathrm{~dB}$

Gain $=4.48 \mathrm{~dB}$

Directivity $=5.66 \mathrm{~dB}$

\subsection{Farfield Directivities and gainsof CNT Star patch with two Superstrate layers}

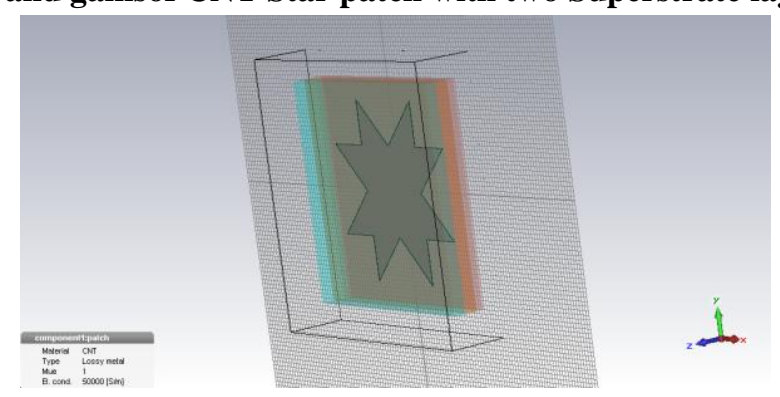

Fig. 14. CNT Star Patch Antenna with two anisotropic Superstrate Layers, $\varepsilon 1_{x x}=9.4 ; \varepsilon 1_{z z}=11.6$ ( layer 1) and $\varepsilon 2_{x x}=13 ; \varepsilon 2_{z z}=10.6$ ( layer 2$)$ 
Table 5. Directivities and gains of CNT Star Patch Antenna with two Anisotropic Superstrate Layers, $\varepsilon 1_{x x x}=$ $9.4 ; \varepsilon 1_{z z}=11.6($ layer 1$)$ and $\varepsilon 2_{z x}=13 ; \varepsilon 2_{z z}=10.6($ layer 2$)$.

\begin{tabular}{|c|c|c|}
\hline F & $\begin{array}{c}\text { Directivity } \\
\mathrm{dBi}\end{array}$ & $\begin{array}{c}\text { Gain } \\
\mathrm{dBi}\end{array}$ \\
\hline $\mathrm{GHz}$ & 3.93 & 3.37 \\
\hline 4.5 & 4.31 & 3.11 \\
\hline 5.5 & 4.39 & 3.61 \\
\hline 7.5 & 4.65 & 3.87 \\
\hline 9.5 & 3.97 & 2.95 \\
\hline 11.5 & 3,97 & 2.64 \\
\hline 13.5 & 7.72 & 4.78 \\
\hline 15.5 & 4.98 & 3.29 \\
\hline 17.5 & 13.2 & 8.56 \\
\hline 19.5 & 14.8 & 8.2 \\
\hline 19.75 & & \\
\hline
\end{tabular}

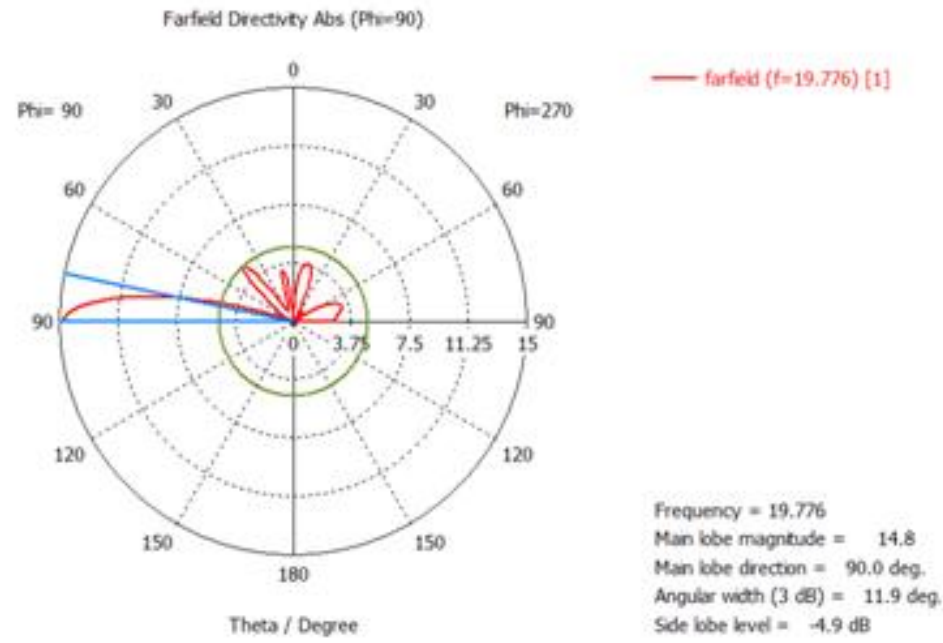

Fig. 15. Directivity of CNT Star Patch at F=19.75 GHz with two Anisotropic Superstrate Layers, $\varepsilon 1_{x x}=9.4$; $\varepsilon 1_{z z}=11.6($ layer 1$)$ and $\varepsilon 2_{x x}=13 ; \varepsilon 2_{z z}=10.6($ layer 2$)$.
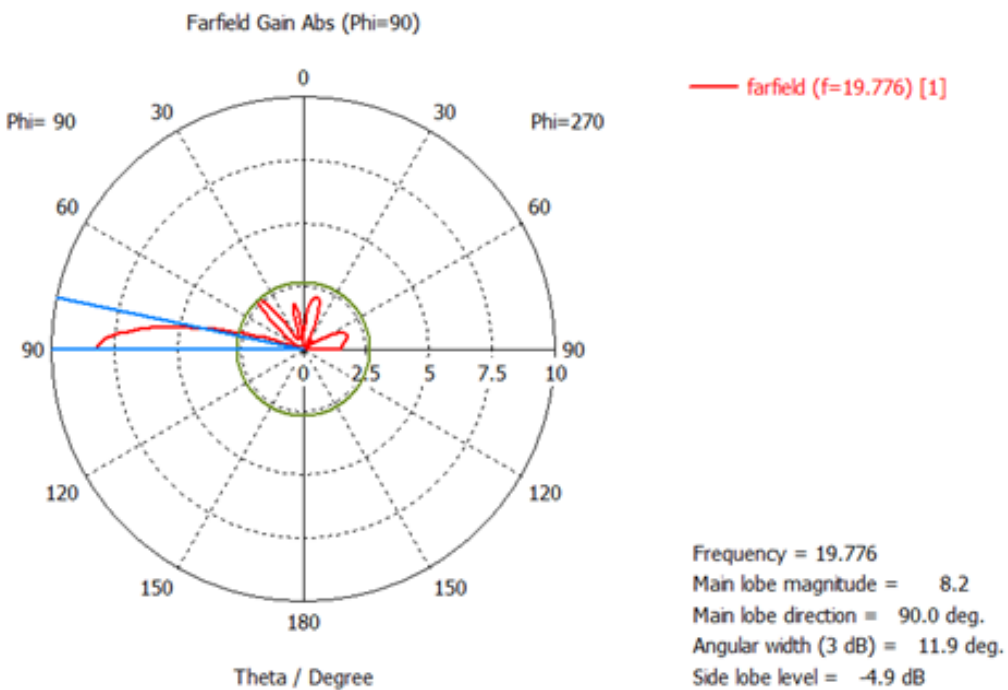

Fig. 16. Gain of CNT Star Patch at $\mathrm{F}=19.75 \mathrm{GHz}$ with two Anisotropic Superstrate Layers, $\varepsilon 1_{z x}=9.4 ; \varepsilon 1_{z z}=$ 11.6( layer 1 ) and $\varepsilon 2_{x x}=13 ; \varepsilon 2_{z z}=10.6($ layer 2$)$. 


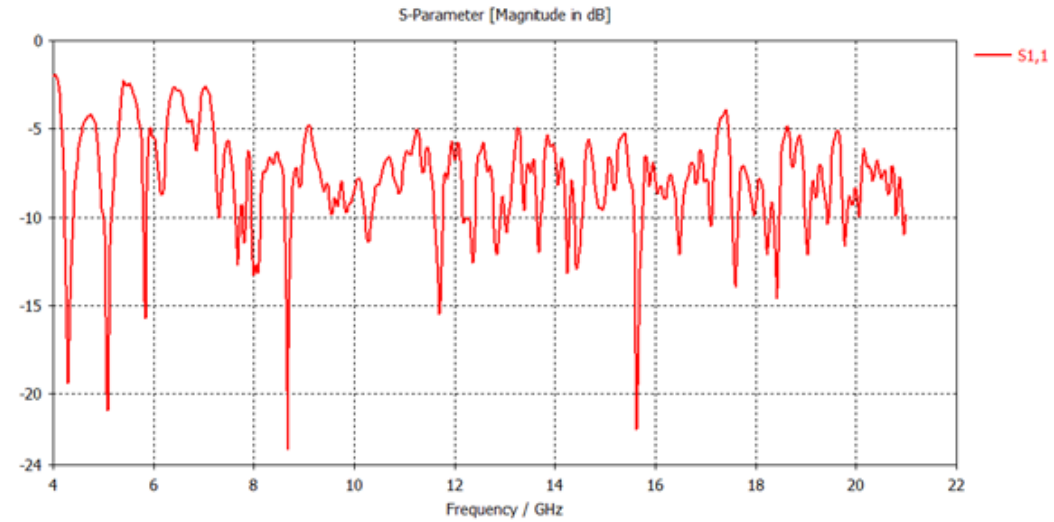

Fig. 17. S11 of CNT Star Patch Antenna with two Anisotropic Superstrate Layers, $\varepsilon 1_{x x}=9.4 ; \varepsilon 1_{z z}=11.6$ ( layer 1) and $\varepsilon 2_{x x}=13 ; \varepsilon 2_{z z}=10.6($ layer 2$)$.

Table 6, S11, Axial Ratio, Gain and Directivities for best Design of CNT Star Patch Antenna with two Anisotropic Superstrate Layers, $\varepsilon 1_{x x}=9.4 ; \varepsilon 1_{z z}=11.6$ ( layer 1 ) and $\varepsilon 2_{x x}=13 ; \varepsilon 2_{z z}=10.6($ layer 2 ).

\begin{tabular}{|c|c|c|c|c|}
\hline $\begin{array}{c}\text { Frequency } \\
\text { GHz }\end{array}$ & $\begin{array}{c}\text { S11 } \\
\text { dB }\end{array}$ & $\begin{array}{c}\text { Axial Ratio } \\
\text { dB }\end{array}$ & $\begin{array}{c}\text { Gain } \\
\text { dB }\end{array}$ & $\begin{array}{c}\text { Directivity } \\
\text { dB }\end{array}$ \\
\hline 4.289 & -19.39 & 19.466 & 3.27 & 4.56 \\
\hline 5.088 & -20.93 & 1.105 & 2.98 & 4.36 \\
\hline 5.836 & -15.697 & 1.092 & 3.98 & 5.42 \\
\hline 7.672 & -12.699 & 1.05 & 1.15 & 1.49 \\
\hline 7.808 & -11.45 & 1.654 & 2.21 & 2.99 \\
\hline 7.995 & -13.29 & 1.246 & 2.67 & 3.62 \\
\hline 8.675 & -23.14 & 1.236 & 0.722 & 1.61 \\
\hline 10.273 & -11.4 & 1.43 & 3.31 & 4.18 \\
\hline 11.701 & -15.5 & 1.374 & 10.3 & 14 \\
\hline 12.364 & -12.57 & 1.46 & 6.07 & 8.88 \\
\hline 12.84 & -12.08 & 1.118 & 4.48 & 6.6 \\
\hline 13.027 & -10.84 & 1.1657 & 1.72 & 2.69 \\
\hline 13.673 & -11.97 & 2.06 & 3.73 & 5.99 \\
\hline 14.251 & -13.19 & 1.27 & 4.15 & 6.16 \\
\hline 14.438 & -12.92 & 1.11 & 5.29 & 7.48 \\
\hline 15.628 & -21.999 & 1.058 & 5.64 & 8.27 \\
\hline 16.478 & -12.07 & 1.59 & 2.68 & 3.92 \\
\hline 17.107 & -10.46 & 1.236 & 2.75 & 3.8 \\
\hline 17.6 & -13.92 & 1.244 & 1.85 & 2.79 \\
\hline 18.224 & -12.106 & 1.44 & 1.04 & 1.63 \\
\hline 18.416 & -14.59 & 1.125 & 3.41 & 5.29 \\
\hline 19.028 & -12.106 & 1.135 & 2.33 & 3.42 \\
\hline 19.436 & -10.35 & 1.57 & 3.94 & 6.5 \\
\hline 19.776 & -11.6 & 1.22 & 1.03 & 1.85 \\
\hline 20.949 & -1.994 & 1.22 & 7.12 & 11.5 \\
\hline
\end{tabular}

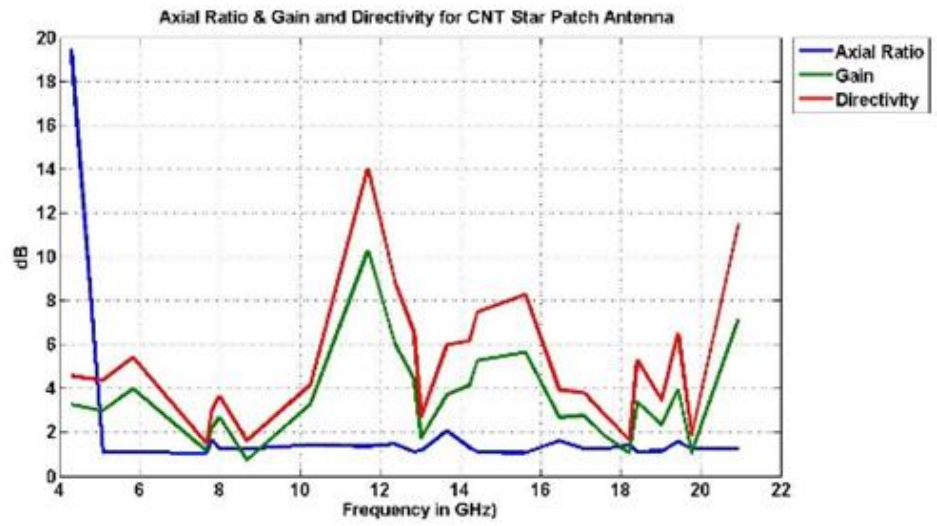

Fig. 18, Axial Ratio, Gain and Directivities for best Design of CNT Star Patch Antenna with two Anisotropic Superstrate Layers, $\varepsilon 1_{x x}=9.4 ; \varepsilon 1_{z z}=11.6$ (layer 1 ) and $\varepsilon 2_{x x}=13 ; \varepsilon 2_{z z}=10.6$ (layer 2).) 
In this configuration, sapphire $\left(\varepsilon_{x x}=9.4 ; \varepsilon_{z z}=11.6\right)$, for Superstrate 1 is used. While), Epsilam-10 $\left(\varepsilon_{x x}=13\right.$; $\varepsilon_{z z}=10.6$ ).is used for superstrate 2. From Fig. 15. Directivity was increased to $14.8 \mathrm{dBi}$, and the gain was 8.2 at frequency fr $=19.75 \mathrm{GHz}$

From Table 6 and Fig. 18, the best Design parameters are :

$\mathrm{F}=11.701 \mathrm{GHz}$

$\mathrm{S} 11=-15.5 \mathrm{~dB}$

Axial Ratio $=1.374 \mathrm{~dB}$

Gain $=10.3 \mathrm{~dB}$

Directivity $=14 \mathrm{~dB}$

\section{Conclusion}

This paper shows a high gain and directivity for CNT Star Patch Antenna embedded in multilayers anisotropic media. Also, it shows, how the anisotropic phenomena can improve the performance of the patch antenna.

\section{References}

[1] B. Braaten, R. Nelson, I. Rogers, Input Impedance and Resonant Frequency of a Printed Dipole With Arbitrary Length Embedded in Stratified Uniaxial Anisotropic Dielectrics,IEEE Antennas and Wireless Propagation Letters. 8, 2009, 806-810.

[2] C. Gürelm, E. Yazga, Characteristics of a Circular Patch Microstrip Antenna on Uniaxially Anisotropic Substrate,IEEE Trans. on Antennas and Propagation, 52 (10), October, 2004, 2532-2537.

[3] F. Bouttout,F. Benabdelaziz, A. Benghalia, D. Khedrouche, T. Fortaki, Uniaxially anisotropic substrate effects on resonance of rectangular microstrip patch antenna, Electronics Letters, 35 (4), 18th February, 1999, 255-256.

[4] R. Nelson, D. Rogers, D'assungao, Resonant Frequency of a Rectangular Microstrip Patch on Several Uniaxial Substrates, IEEE Trans. on Ante.Propagation, 38 (7), July, 1990, 973-981.

[5] A. Eroglu, J. K. Lee, Far field radiation from an arbitrarily orientated Hertzian dipole in the presence of layered anisotropic medium,IEEE Trans. Antennas Propag., 53 (12),December, 2005, 3963-3973.

[6] L. Yang, A. Rida, R. Vyas, and M. M. Tentzeris.(2007). RFID tag and RF structures on a paper substrate using inkjet-printing technology. IEEE Trans. Microw. Theory Tech., vol. 55, no. 12, 2894-2901.

[7] P. V. Nikitin, S. Lam, and K. V. S. Rao.( 2005). Low cost silver ink RFID tag antennas. inProc. IEEE Antennas Propag. Society Int. Symp., 353-356.

[8] J. Anguera, J.-P. Daniel, C. Borja, J. Mumbru, C. Puente, T. Leduc, N. Laeveren, and P. V. Roy.(2008). Metallized foams for fractal-shaped Microstrip antennas. IEEE Antennas Propag. Mag., vol. 50, no. 6. 20-38

[9] V. Losada, R. Boix, \& M. Horno, " Full-Wave Analysis of Circular Microstrip Resonators in Multilayered Media Containing Uniaxial Anisotropic Dielectrics, Magnetized Ferrites, and Chiral Materials. IEEE Trans. on Microwave Theory and Techniques, Vol. 48, No. 6, 1057-1064.

[10] P. Avouris. (2002). Carbon nanotube electronics. Chem. Phys., vol. 281, 429-445

[11] G. W. Hanson. (2005). Fundamental transmitting properties of carbon nanotube antennas. IEEE Trans. Antennas Propag., vol. 53, no. $11,3426-3435$

[12] G. W. Hanson. (2006). Current on an infinitely-long carbon nanotube antenna excited by a gap generator. IEEE Trans. Antennas Propag., vol. 54, no. 1, 76-81

[13] M. Gharbi, A. Affandi, S. Ali, S. Application of The Moment Method in The Spectral Domain,Life Science Journal, 5,2015,1 23.

[14] A. Affandi, M. Gharbi, S. Ali, Plane Wave Spectral Integral Representation of the Dyadic Greens Function of Layered Media, Life Sci. Journal, 4, 2015,164-174. 